\title{
Optical response of gold and upconversion nanoparticles assembled via DNA interaction
}

De Fazio, Angela, Haines, Jack, Courtier, Alex, Muskens, Otto, Kanaras, Antonios

Angela F. De Fazio, Jack Haines, Alex Courtier, Otto L. Muskens, Antonios G. Kanaras, "Optical response of gold and upconversion nanoparticles assembled via DNA interaction," Proc. SPIE 10892, Colloidal Nanoparticles for Biomedical Applications XIV, 108920A (7 March 2019); doi: $10.1117 / 12.2504970$

SPIE. Event: SPIE BiOS, 2019, San Francisco, California, United States 


\title{
Optical response of gold and upconversion nanoparticles assembled via DNA interaction
}

\author{
Angela F. De Fazio ${ }^{\mathrm{a}}$, Jack Haines ${ }^{\mathrm{a}}$, Alex Courtier ${ }^{\mathrm{a}}$, Otto L. Muskens ${ }^{\mathrm{a}, \mathrm{b}}$ * , Antonios G. Kanaras $^{\mathrm{a}, \mathrm{b}, *}$ \\ a School of Physics and Astronomy, University of Southampton, Southampton, UK, SO171BJ \\ bInstitute for Life Sciences, University of Southampton, Southampton, UK, SO171BJ \\ *Email: a.kanaras@soton.ac.uk ,o.muskens@soton.ac.uk phone: 00442380592466, fax: (+44) (0) 2380593910
}

\begin{abstract}
In this paper we present an optical characterization of nanoparticle assemblies containing plasmonic gold nanoparticles and fluorescent upconversion nanoparticles in different ratios. Both the two-photon luminescence derived from gold nanoparticles and the fluorescence originated from upconversion nanoparticles are detected. The optical signatures reflect the composition of the assemblies.
\end{abstract}

Keywords: nanoparticles, gold, upconversion, fluorescence, two photon photoluminescence

\section{INTRODUCTION}

In the last two decades, nanoparticles (NPs) have been increasingly studied for their unique properties arising from their size, shape, and chemical composition ${ }^{1,2-8}$. Among various types of nanoparticles, gold nanoparticles (AuNPs) have attracted particular interest thanks to their ease of synthesis and their optical, electronic and thermal properties, which enable their application in different fields ranging from biology to nanophotonics ${ }^{9-12}$.

Recently, lanthanide-doped NPs have also been employed because of their ability to absorb infrared light and emit photons at higher energies resulting from an internal upconversion process. These upconversion nanoparticles (UCNPs) have superior optical properties, such as sharp emission lines, variable color emission through compositional tuning, long luminescence lifetimes and prolonged photostability ${ }^{13,14}$, all of which represent promising characteristics for energy applications, sensing, multimodal imaging and photodynamic therapy ${ }^{13,15}$. The coupling of particles with plasmonic and fluorescent properties represents an effective way towards hybrid materials with different optical properties ${ }^{16-19}$. Amongst the various approaches to effectively achieve the coupling of particles, deoxyribonucleic acid (DNA) represents a unique tool for their precise arrangement due to its selectivity that allows two complementary DNA strands to form a DNA doublehelix. The driving force of this binding is the maximization of the hydrogen bonds between the DNA nucleotide bases (adenine, thymine, guanine, and cytosine), which is achieved when a thymine binds an adenine in the opposite strand, and a guanine couples with a cytosine. DNA strands, or oligonucleotides, will bind in a duplex in such a way to maximize the number of complementary paired bases. This property of DNA has been extensively used in chemistry and biology to assemble nanoparticles together ${ }^{20-27}$.

In this paper, we present the assembly of gold and upconversion nanoparticles and a first explorative study of their optical properties as a function of different mixing ratios of the two components. Our study focuses on two characteristic signatures of the individual systems, namely upconversion fluorescence of UCNPs and two-photon photoluminescence (TPPL) from the gold nanoparticles. Both processes can be independently addressed by selecting the wavelength and emission characteristics of the excitation light source. Upconversion is a process that relies on energy transfer between lanthanide ions that are photoexcited by a narrowband laser. The excitation laser wavelength of $980 \mathrm{~nm}$ overlaps with the energy levels of $\mathrm{Yb}^{3+}$ and $\mathrm{Er}^{3+}$ ions. The upconversion mechanism is mediated through slow (microsecond) internal carrier 
dynamics and energy transfer between long-lived electronic states and hence continuous wave excitation is efficient in producing a sizeable upconversion signal. In contrast, TPPL requires ultrafast laser pulses to achieve a measurable twophoton cross section, as this is mediated through short-lived (virtual) states in the gold nanoparticle. The TPPL is excited at a slightly longer wavelength of $1060 \mathrm{~nm}$ which is sufficiently far away from the lanthanide energy levels to prevent direct excitation of UCNP upconversion. The study presented here shows the first steps in achieving a quantitative analysis of these optical processes in the hybrid system.

\section{MATERIALS AND METHOD}

\subsection{Materials}

All reagents were purchased from Sigma Aldrich throughout and used without further purification. Oligonucleotides were purchased from ATDBio. All glassware and stirrer bars were rinsed with Aqua Regia followed by Milli-Q water. Commercially available reagents and solvents were purchased from Sigma-Aldrich and used without further purification. Assembly of the DNA-NPs aggregates was performed in an Applied BioSystem ProFlexTM PCR System thermal cycler.

\subsection{Synthesis of gold nanoparticles}

A boiling solution of sodium citrate $(5 \mathrm{~mL}, 2 \% \mathrm{wt})$ was added to a boiling solution of sodium tetrachloroaurate (100 mL, $1 \mathrm{mM}$ ) under vigorous stirring. A color change occurred from yellow through to colorless to purple and finally dark through to wine red. Once the red color was obtained, the boiling and the stirring were carried for 15 additional minutes. Then, the reaction mixture was left to cool down at room temperature under stirring. Then, particles were purified through two sets of centrifugation at $5000 \mathrm{rpm}$ and re-dissolved in a minimum amount of Milli-Q water. This suspension was stored at $4^{\circ} \mathrm{C}$ prior to further use.

\subsection{DNA attachment on gold nanoparticles}

In a typical experiment, citrate-coated AuNPs $(10 \mathrm{pmol}, 500 \mu \mathrm{l})$ were incubated overnight in phosphate buffer $(200 \mu \mathrm{l}$, $0.1 \mathrm{M}$ ) together with a single-stranded DNA (ssDNA, $800 \mathrm{pmol}, 40 \mu \mathrm{l}$ ) (see Table 1) modified with a thiol termination at the 3' end. Six additions of a $\mathrm{NaCl}(106 \mu \mathrm{l}, 2 \mathrm{M})$ solution over a period of $8 \mathrm{~h}$ were introduced to achieve a final salt concentration of $0.3 \mathrm{M}$. The resulting ssDNA-coated AuNPs were purified by three subsequent centrifugation (16400 rpm, $10 \mathrm{~min}$ ) steps. Finally, the conjugates were stored in Milli-Q water at $4{ }^{\circ} \mathrm{C}$.

Table 1 DNA sequences used for the functionalization of gold and upconversion nanoparticles.

\begin{tabular}{|l|l|}
\hline Name & Single stranded DNA sequence \\
\hline Oligo1 & 5'-TAC TTC CAA TCC AAT TTT TTT TTT TTT TTT - 3'thiol \\
\hline Oligo2 & 5'-ATT GGA TTG GAA GTA TTT TTT TTT TTT TTT -3'thiol \\
\hline Oligo2b & 5'-ATT GGA TTG GAA GTA TTT TTT TTT TTT TTT -3'amino \\
\hline
\end{tabular}

\subsection{Synthesis of $\mathrm{NaYF}_{4}: \mathrm{Yb}^{+3}(20 \%), \mathrm{Er}^{+3}(2 \%)$ nanoparticles}

Lanthanide upconversion nanoparticles were synthesized by minor modifications to a previously reported thermal decomposition protocol ${ }^{5}$. The crystalline matrix was created by using yttrium(III) chloride hexahydrate (236.62 mg, 0.78 mmol,). Ytterbium(III) chloride hexahydrate (77.5 mg, $0.20 \mathrm{mmol}$,) was used as the sensitizer and erbium(III) chloride 
hexahydrate (7.63 mg, $0.02 \mathrm{mmol}$,) as emitter. The ratio of matrix/sensitizer/emitter was kept as 78\%:20\%:2\%. All the salts were dissolved with oleic acid (6 mL, $19 \mathrm{mmol}$ ) ) and 1-octadecene $(15 \mathrm{~mL}, 46.9 \mathrm{mmol})$ in a three-necked roundbottom flask. The flask was placed in a heating mantle equipped with a temperature probe and heated at $160^{\circ} \mathrm{C}$ for $1 \mathrm{~h}$ and 30 min under argon flow to allow the solubilization of the salts. Then, the solution was cooled down to room temperature and a solution of methanol $(10 \mathrm{ml})$ containing sodium hydroxide $(100 \mathrm{mg}, 2.5 \mathrm{mmol})$ and ammonium fluoride $(148.16 \mathrm{mg}$, $4 \mathrm{mmol}$ ) was added dropwise to the reaction under vigorous stirring. This mixture was slowly heated to $110^{\circ} \mathrm{C}$ for $2 \mathrm{~h}$ under argon gas at atmospheric pressure and then 30 more minutes under vacuum. Next, the temperature was raised to $310^{\circ} \mathrm{C}$ and left to react for $1 \mathrm{~h}$ and $10 \mathrm{~min}$. Subsequently, the mixture was left to cool to room temperature, and the $\mathrm{NaYF}_{4}: \mathrm{Yb}^{+3}, \mathrm{Er}^{+3}$ nanoparticles were collected by precipitation in ethanol and centrifugation (8000 rpm, $\left.10 \mathrm{~min}\right)$. The supernatant was removed and the pellet redispersed in ethanol and centrifuged again (8000 rpm, $15 \mathrm{~min}$ ). This process was repeated three times, after which the precipitate was left to dry overnight. The weight of the resulting nanoparticles was measured, and the particles $(10 \mathrm{mg} / \mathrm{ml})$ were stored in hexane at room temperature until further use.

\subsection{DNA attachement on $\mathrm{NaYF}_{4}: \mathrm{Yb}^{+3}(20 \%) \mathrm{Er}^{+3}(2 \%)$ nanoparticles}

The functionalization of oligonucleotides around the previously synthesized UCNPs was achieved via a three-step procedure, comprising of i) silanization of upconversion nanoparticles, ii) carboxylation of the nanoparticle surface and finally iii) coupling of amine-modified oligonucleotides via EDC/NHS chemistry.

The growth of a silica shell was performed via a reverse micro-emulsion route. Specifically, IGEPAL CO-520 (500 mg) was dissolved in $10 \mathrm{~mL}$ of hexane containing $40 \mathrm{mg}$ of UCNPs and mixed via sonication. Then, an ammonium hydroxide solution (100 $\mu 1,30 \% \mathrm{v} / \mathrm{v}$,) was added to the particles and the solution was sonicated for 5 minutes. Finally, tetraethyl orthosilicate $(75 \mu \mathrm{l})$ was injected under vigorous stirring. After $12 \mathrm{~h}$, the silica-coated NPs were collected by precipitation from ethanol and washed by three rounds of centrifugation in ethanol ( $8000 \mathrm{rpm}, 15 \mathrm{~min}$ ). Then, amination was conducted by adding the amino enriched silane reagent APTMS $(150 \mu \mathrm{l}, 0.68 \mathrm{mmol})$ to the silica coated UCNPs dissolved in $10 \mathrm{ml}$ of ethanol. The APTMS reacts with the Si-O on the surface of the silica shell to covalently attach an amino group to the surface of the particles. The mixture was stirred (500 rpm, RT) overnight and purified in ethanol via centrifugation (8000 rpm, $15 \mathrm{~min}, 3 \mathrm{x})$. The resultant pellet was re-dispersed in $5 \mathrm{ml}$ of DMF for further functionalization. For the creation of carboxy-terminations on the surface of the UCNPs, succinic anhydride $(150 \mathrm{mg}, 1.49 \mathrm{~nm})$ were added dropwise to the previously obtained UCNPs- $\mathrm{NH}_{2}$ and let to react overnight under a blanket of argon. Purification was performed by three rounds of centrifugation in ethanol (8000 rpm, $15 \mathrm{~min}$ ), after which the particles were dispersed in borate buffer for the coupling reaction with amine-modified oligonucleotides.

1-Ethyl-3-(3-dimethylaminopropyl)carbodiimide (EDC $0.3 \mathrm{M}, 20 \mu \mathrm{l}$ ) in MES buffer and sulfo-N-hydroxysuccinimide (NHS, $0.3 \mathrm{M}, 40 \mathrm{mg}$ ) also in MES buffer were added to the COOH-UCNPs $(2 \mathrm{mg} / \mathrm{ml}, 1 \mathrm{ml})$ in borate buffer, and let to stir for $30 \mathrm{~min}$, after which, an excess of the desired amino-terminated oligonucleotide was added to the mixture (details of the sequences in Table 1), and left to react overnight. Three rounds of centrifugation in water (16400 rpm, $30 \mathrm{~min})$ were carried out to purify the DNA-UCNPs, which were dispersed in $1 \mathrm{ml}$ of MilliQ water for storage.

\subsection{Assembly of gold and upconversion nanoparticles}

The assembly of the DNA-NPs was performed by heating two batches of NPs decorated with complementary DNA strands previously incubated in $0.3 \mathrm{M} \mathrm{NaCl}$ phosphate buffer solution at $70^{\circ} \mathrm{C}$ (above the melting temperature of the DNA duplex) and slowly cooled down to room temperature using a programmable thermal cycler (rate $0.1^{\circ} \mathrm{C} / 10 \mathrm{mins}$ ). Nanoparticle assemblies were sedimented at the bottom of the tube, the supernatant solution was removed and $200 \mu \mathrm{l}$ of $\mathrm{NaCl} 0.3 \mathrm{M}$ in phosphate buffer was added for storage.

\subsection{Characterization of nanoparticles}

UV - visible spectra of colloidal gold nanoparticles to determine their concentration were collected using Cary $100 \mathrm{UV}$ Vis spectrophotometer over the range from 200 to $800 \mathrm{~nm}$. TEM images were obtained on a Tecnai transmission electron microscope operating at a bias voltage of $75 \mathrm{kV}$. All samples ( $\sim \mu \mathrm{L}$ droplets of them) were deposited and evaporated on Carbon Film 400 Mesh Copper grids. Zeta potentials were measured on a Malvern Zetasizer Nano ZS with a He-Ne light source (633 nm wavelength). Each measurement was performed with a $173^{\circ}$ backscattering arrangement, consisted of 25 sub-runs and was repeated three times. 


\section{RESULTS AND DISCUSSION}

\subsection{Nanoparticles synthesis and functionalization}

While the functionalization of gold nanoparticles with oligonucleotides is an easy and well-established protocol, the coating of upconversion nanoparticles with oligonucleotides is a considerably more elaborate process, which requires multiple steps before the actual DNA attachment on the UCNPs. Initially the upconversion nanoparticles are coated with a thin shell of silica ${ }^{28}$. Figure 1 shows a TEM image of these nanoparticles where the thin shell of silica is observed as it is of lower contrast in comparison to the nanoparticle core. The size distribution of the silica-coated nanoparticles and zeta potential measurements are also presented. As expected, a negative potential is recorded for the silanized UCNPs (-40 $\mathrm{mV})$, which became less negative after the amination step $(-4 \mathrm{mV})$. The zeta potential value after the carboxylation step was again strongly negative $(-32 \mathrm{mV})$.
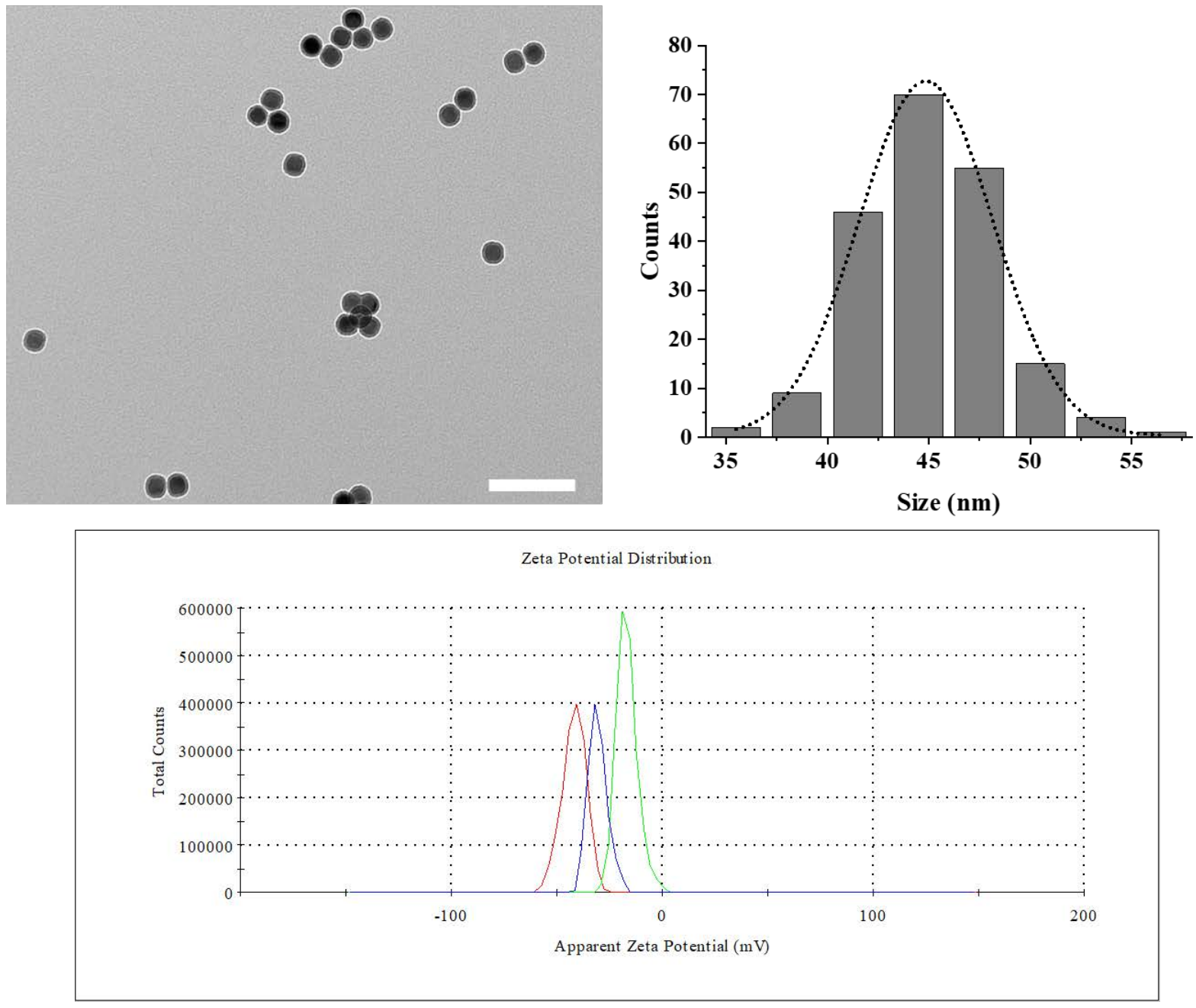

Figure 1 (Top left) Transmission Electron Micrograph of silanized UCNPs, scale bar $200 \mathrm{~nm}$. (Top right) Corresponding size distribution histogram. (Bottom) Zeta potential measurements for UCNPs-SiO2 (red curve), UCNPs-NH2 (green curve) and UCNPs$\mathrm{COOH}$ (blue curve).

To prove the efficacy of the DNA functionalization in assembling the particles together, the melting profile for a mixed assembly of AuNPs@Oligo1 and UCNPs@Oligo2b 1/1 was recorded. The melting analyses relies on the DNA hyperchromicity properties. In more details, single stranded DNA (ssDNA) has a strong absorption at $260 \mathrm{~nm}$, and its 
intensity sharply decreases for double-stranded DNA (dsDNA). By heating up, the DNA duplex denatures into two ssDNA with stronger optical density, and the process can be followed by UV-Visible spectroscopy. In Figure 2, the melting curve obtained by continuously recording the absorbance at $260 \mathrm{~nm}$ is reported, showing the typical sigmoidal shape expected for hypercromicity. Furthermore, the melting temperature (the temperature value at which $50 \%$ of the dsDNA is denatured) is considerably higher than what predicted for DNA duplex not attached to any particles $\left(80^{\circ} \mathrm{C}\right.$ instead of $\left.49^{\circ} \mathrm{C}\right)$. This is in agreement with what expected when an large assembly of nanoparticles loaded with a dense shell of DNA is under analysis, as a markedly higher stability is attributed to cooperative effects ${ }^{29}$.

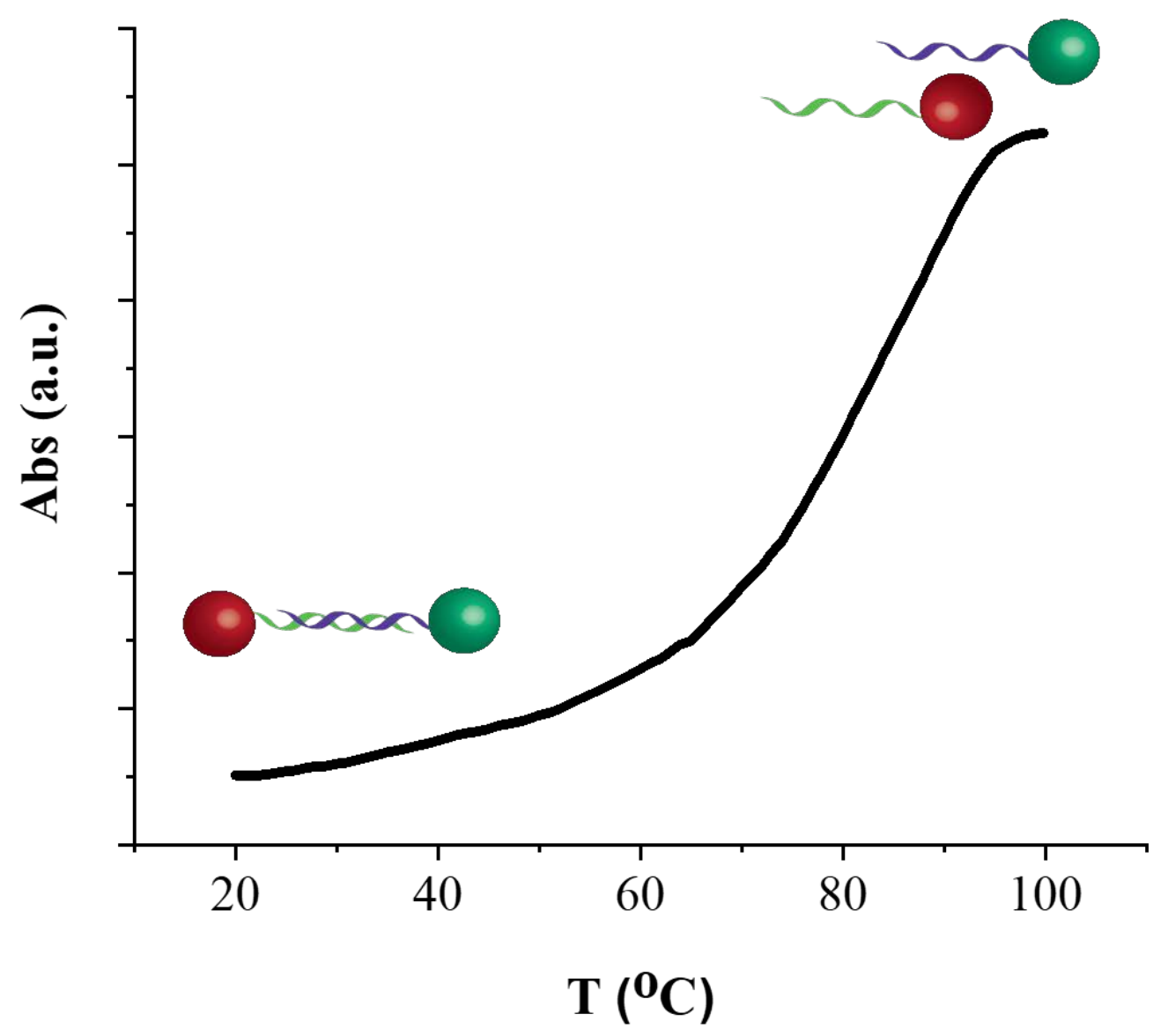

Figure 2 DNA melting curve of an assembly of gold and upconversion nanoparticles.

\subsection{Optical characterization of mixed gold and upconversion aggregates}

Two-photon photoluminescence (TPPL) is a well-known process for gold nanoparticles ${ }^{30}$. This phenomenon involves the absorption of two photons of comparable energy to excite a gold nanoparticle. Each photon carries approximately half the energy required to excite the particle. Since the probability of near simultaneous absorption of two photons is extremely low, a femtosecond (fs) pulsed laser is therefore used, which provides a high flux of photons.

The TPPL setup employed includes a Yb-doped fibre laser (Fianium) with emission centered at $1060 \mathrm{~nm}$, pulse duration of $11 \mathrm{ps}$, and a laser power of around $30 \mathrm{~mW}$. The laser beam is directed using a galvo scanning mirrors and is focused onto the sample using a 60x objective lens. The sample holder was a 18-wells micro-slide. The nanoparticle assemblies are located by bright field imaging and the measurement is performed by scanning the laser beam on the so-identified area thanks to a galvo mirror. The emitted signal is finally collected on an avalanche photodetector (Excelitas SPCM-AQRH). 
Photon counts were collected as a function of position using a laser-scanning arrangement and a data-acquisition system (Becker\&Hickl). The setup includes a $1050 \mathrm{~nm}$ long pass filter to exclude the signal emitted by the UCNPs.

Photon upconversion is a non-linear process consisting in the sequential absorption of two lower energy photons, which are subsequently emitted at a higher energy wavelength. Upconversion nanoparticles are inorganic crystalline structures composed by a matrix (typically $\mathrm{NaYF}_{4}$ ) hosting rare-earth elements, which act as sensitizers and activators for the upconversion of infrared photons. Usually, sensitizers are chosen so that they possess relatively large photo-absorption cross-sections and appropriate energy levels to match the corresponding activators. These characteristics are well-met by lanthanide ions, due to the high number of energy levels in the $4 \mathrm{f}-5 \mathrm{~d}$ energy levels, which provide multiple energy transfer pathways for upconversion emission. Ytterbium $\mathrm{Yb}^{3+}$ is a highly efficient photo-sensitizer with high absorption at $976 \mathrm{~nm}$ (transition ${ }^{2} \mathrm{~F}_{7 / 2} \rightarrow{ }^{2} \mathrm{~F}_{5 / 2}$ ). The absorbed energy is then transferred to an activator within the matrix (such as $\mathrm{Er}^{3+}$ ), which can then emit the energy in the visible spectrum ${ }^{31,32}$.

The upconversion fluorescence was performed in a manually aligned setup with a continuous wave $980 \mathrm{~nm}$ diode laser (Thorlabs) as excitation source with power of $10 \mathrm{~mW}$, and was collected by the avalanche photodetector in the same way as the TPPL. In Figure 3 a schematic drawing of the TPPL/Fluorescence setup is reported.

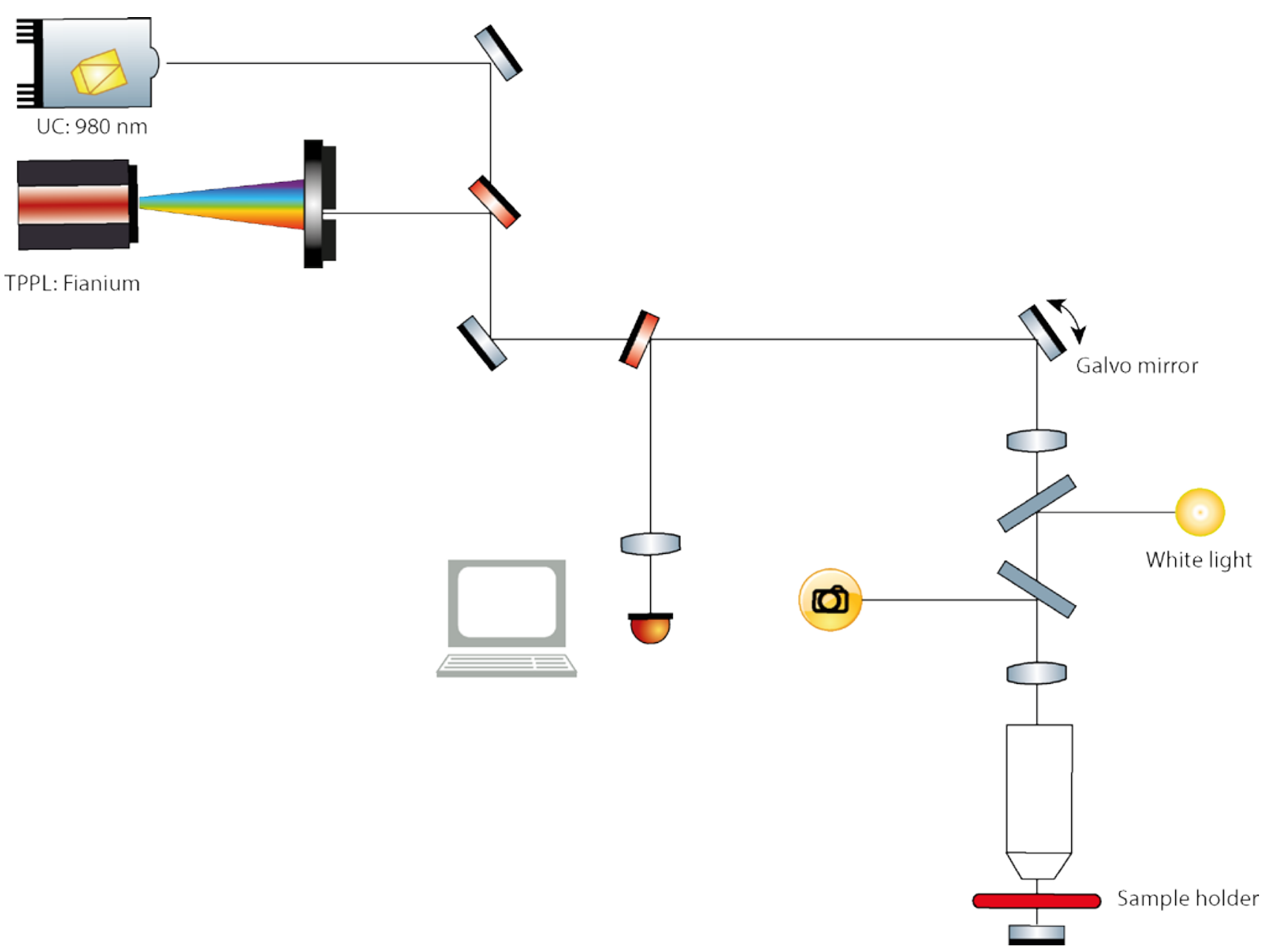

Figure 3. Schematic diagram of the laser setup built for measuring the upconversion fluorescence and the two photon photoluminescence of the nanoparticle assemblies. Two different laser sources are directed through a series of mirrors into the objective and up to the sample. 


\subsection{Mixed assemblies imaging and comparison}

AuNPs functionalized with a shell of Oligo1 were mixed in different ratios with AuNPs functionalized with the complementary strands Oligo2 and UCNPs functionalized with Oligo2b. The binding sequence of Oligo2 and Oligo2b is the same as it was design to be complementary with Oligo1, the difference between the two ssDNA is in the 3' end modification. For Oligo2 the modification is a thiol because it is meant to attach the gold surface, while it is an amino group for Oligo2b to be coupled with the carboxy termination on the UCNPs surface.

The mixing ratio for the assembly of gold and upconversion nanoparticles is started from the pure gold aggregates containing AuNPs@Oligo1:AuNPs@Oligo2=1:1 and no UCNPs@Oligo2b, the amount of AuNPs@Oligo2 was gradually decreased while UCNPs@Oligo2b increased, to create a competition between the two different kind of nanoparticles bearing the same ssDNA to go and bind with the complementary one, which was kept at a constant concentration. Details of the samples prepared are shown in Table 2.

Table 2 Ratio of gold and upconversion nanoparticles used in assembling the aggregates. In a) a fixed amount of AuNPs@Oligo1 is kept, while the ratio between the competing AuNPs@Oligo2 and UCNPs@Oligo2b.

\begin{tabular}{|c|c|c|}
\hline AuNPs@Oligo1 & AuNPs@Oligo2 & 0 \\
\hline 1 & 1 & 0.25 \\
\hline 1 & 0.75 & 0.5 \\
\hline 1 & 0.5 & 0.75 \\
\hline 1 & 0.25 & 1 \\
\hline 1 & 0 & \\
\hline
\end{tabular}
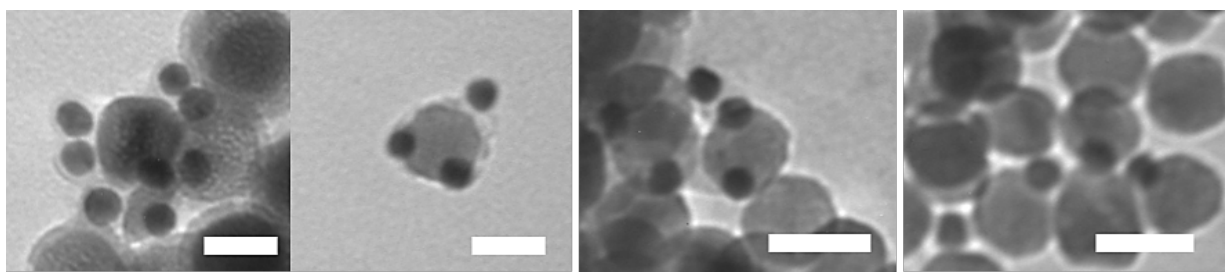

Figure 4 Transmission electron micrographs of UCNPs surrounded by AuNPs satellites. All scale bars $50 \mathrm{~nm}$.

Transmission electron micrographs (Figure 4) were also taken on selected samples prepared by dropcasting a drop of the sample on a carbon coated TEM grid and letting to dry. This process causes the DNA to shrink as the solvent evaporates and therefore it is not an exact representation of the nanoparticles assemblies in a three-dimensional space. Figure $\mathbf{4}$ shows the amorphous aggregates of the AuNPs/UCNPs. The smaller AuNPs appear to arrange around the bigger UCNPs and intercalate them.

The comparison of the signal arising from TPPL or fluorescence was performed by collecting measurement on the same sample area with both the supercontinuum laser for TPPL and the $980 \mathrm{~nm}$ laser for fluorescence. The signal intensity ratio was obtained by summing the intensities of all the pixel in the TPPL measurement and dividing by the sum of all the pixels in the upconversion measurements on the same area, or in symbols: intensity ratio $=\sum$ Pixel intensity TPPL $/ \sum$ Pixel intensity UC.

Figure 5 shows examples of the images used for the pixel analysis. In general, the fluorescence signal had a considerably higher intensity than the corresponding TPPL, which is expected for these two different physical phenomena. In addition to that, in Figure 5 is clearly shown the co-localization of gold and upconversion nanoparticles. 

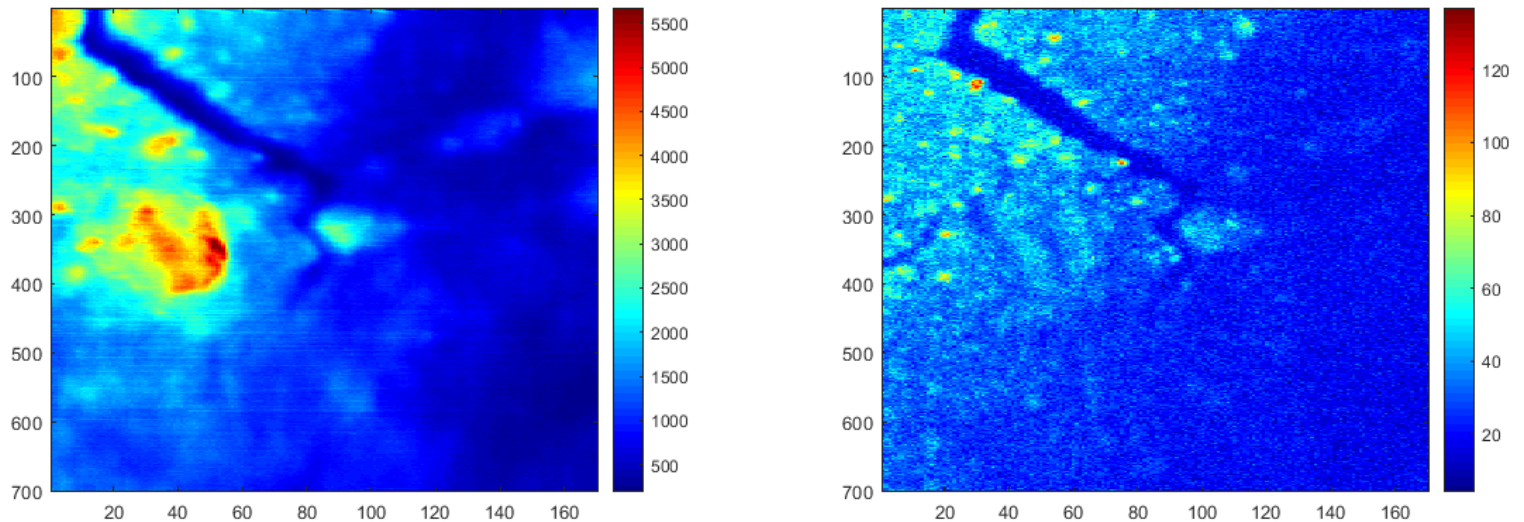

Figure 5 Exemplary images taken on the same area of a mixed assembled sample (AuNPs@Oligo1 plus UCNPs@Oligo2b/AuNPs@Oligo2 0.5/0.5). On the left the fluorescence signal from the UCNPs under excitation at 980 nm. On the right, TPPL from AuNPs excited at $1050 \mathrm{~nm}$, clearly co-localized with the emission of the UCNPs.

Figure 6 displays a graph showing the relationship between the intensity ratio calculated as above and the percentage of AuNPs. More specifically, it shows the data from mixed aggregates containing AuNPs@DNA1 in a fixed amount plus AuNPs@Oligo2 and UCNPs@Oligo2b in varying ratios. A clear pattern is visible from these samples, as the smallest intensity ratio (i.e. the least TPPL signal) corresponds to the sample containing the least amount of gold (AuNPs@Oligo2/UCNPs@Oligo2b=0.25/0.75). At the same time, the sample showing the highest intensity ratio is the one containing more gold (AuNPs@Oligo2/UCNPs@Oligo2b = 0.75/0.25). Consistently, the aggregate made by equivalent amount of AuNPs@Oligo2and UCNPs@Oligo2b (0.5/0.5) lies in the middle between the two other samples.

Our first results show that TPPL and UC can be independently measured on mixed assemblies, yielding a quantitative comparison between the two processes. Further in-depth studies are needed to elucidate any effects of cross-interaction in the hybrid materials system. This will be pursued in follow-on work. Overall, the TPPL intensities are lower than the UC which currently presents some challenges in the analysis at the single-crystal level. While we have used large-area scans at short (ms) pixel dwell times, sensitivity could be greatly increased by investigating individual crystals for longer periods of time. Alternatively, higher excitation intensity and/or a higher numerical aperture objective could be used to increase photon yield. A careful balance is needed between excitation intensity and damage threshold of the system, in particular for prolonged exposure times. It was found in our current work that laser damage did not pose a limitation, however thermal and non-thermal damage can be expected at optical powers exceeding tens of $\mathrm{mW}$, in particular for gold nanoparticles. Other studies of interest could exploit these dependences to investigate heat generation by nanoparticle-assemblies. 


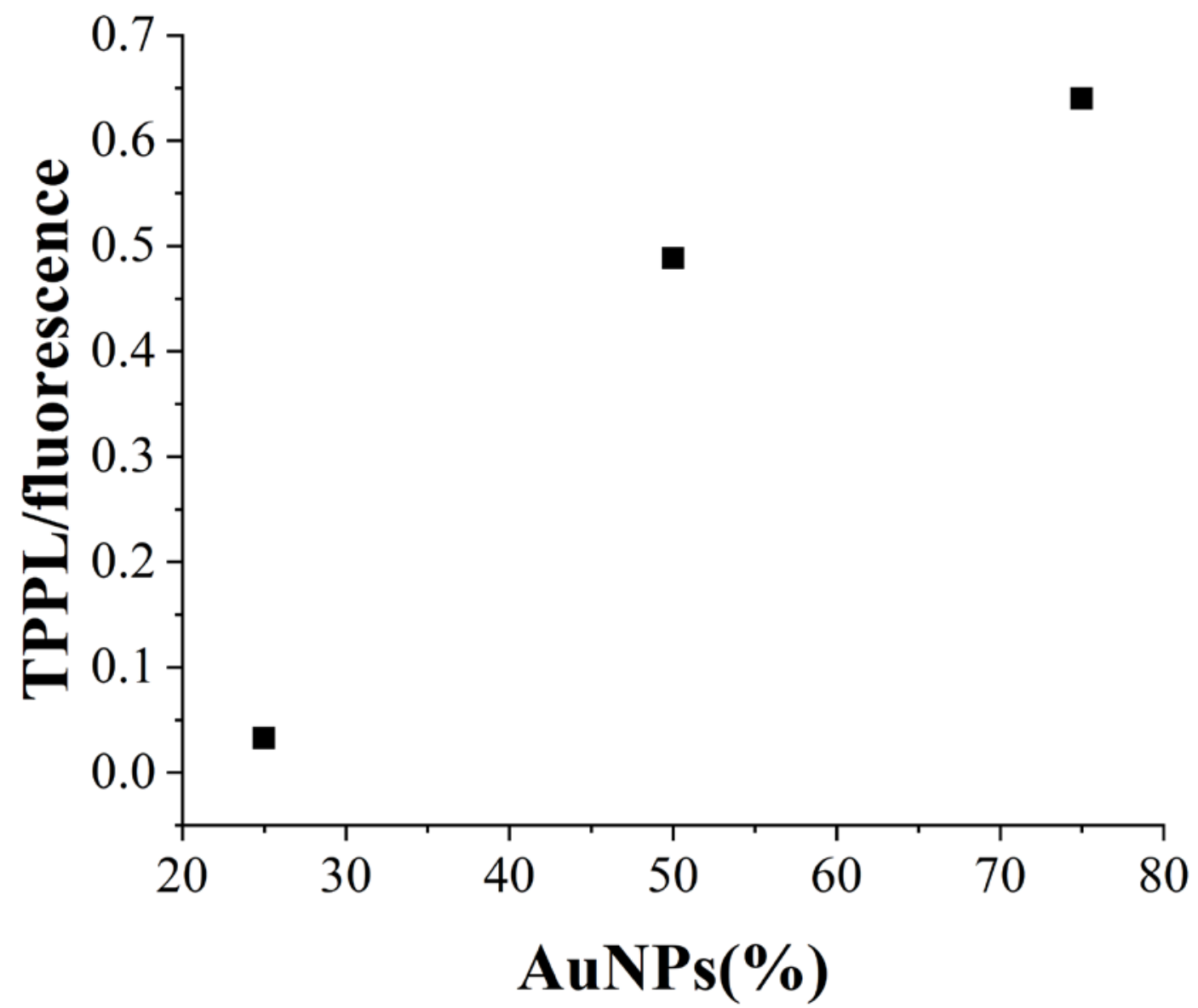

Figure 6. Plot of the ratio between the intensity of TPPL signal and the intensity of fluorescence signal taken on the same sample area, vs the percentage of AuNPs@Oligo2 competing with UCNPs@Oligo2b to bind AuNPs@Oligo1.

\section{CONCLUSIONS}

In conclusion, we have fabricated hybrid assemblies consisting of both gold nanoparticles and upconversion nanoparticles using complementary DNA strands. We functionalized gold nanoparticles and upconversion nanoparticles with DNA strands following either thiol chemistry for the gold nanoparticles or coupling chemistry for the upconversion nanoparticles. These particles were then mixed in different ratios to form assemblies of different upconversion to gold proportions. The various types of these assemblies were characterized optically using two photon photoluminescence and fluorescent measurements. It was found that the two characteristic signatures of two-photon and upconversion emission could be independently addressed using respectively continuous wave and pulsed laser excitation at two different wavelengths. In the future, we aim to expand this line of studies to address the synergy between the two types of particles and their hybrid arrangement and to expand the characterization to crystalline assemblies of DNA-nanoparticles.

\section{ACKNOWLEDGMENTS}

AGK, OM and AFDF would like to acknowledge DSTL for funding of a PhD studentship. 


\section{REFERENCES}

[1] Conde, J., Dias, J.T., Grazú, V., Moros, M., Baptista, P. V, and de la Fuente, J.M., "Revisiting 30 years of biofunctionalization and surface chemistry of inorganic nanoparticles for nanomedicine.," Frontiers in chemistry 2, 48 (2014).

[2] Schmid, G., [Nanoparticles: from theory to application] , in Nanotechnology, G. Schmid, Ed., Wiley-VCH (2004).

[3] Diodati, S., Dolcet, P., Maurizio, C., and Gross, S., "Pursuing the Crystallization of Mono- and Polymetallic Nanosized Crystalline Inorganic Compounds by Low-Temperature Wet- Chemistry and Colloidal Routes,” Chemical Reviews (2015).

[4] Selvan, S.T., Tan, T.T.Y., Yi, D.K., and Jana, N.R., "Functional and multifunctional nanoparticles for bioimaging and biosensing.," Langmuir : the ACS journal of surfaces and colloids 26(14), 11631-41 (2010).

[5] Hu, H., and Zhang, W., "Synthesis and properties of transition metals and rare-earth metals doped ZnS nanoparticles,” Optical Materials 28(5), 536-550 (2006).

[6] Jain, P.K., Huang, X., El-Sayed, I.H., and El-Sayed, M.A., "Noble metals on the nanoscale: Optical and photothermal properties and some applications in imaging, sensing, biology, and medicine," Accounts of Chemical Research 41(12), 1578-1586 (2008).

[7] Hahn, M. a, Singh, A.K., Sharma, P., Brown, S.C., and Moudgil, B.M., "Nanoparticles as contrast agents for invivo bioimaging: current status and future perspectives.," Analytical and bioanalytical chemistry 399(1), 3-27 (2011).

[8] Daniel, M.-C., and Astruc, D., "Gold nanoparticles: assembly, supramolecular chemistry, quantum-size-related properties, and applications toward biology, catalysis, and nanotechnology.,” Chemical reviews 104(1), 293-346 (2004).

[9] Day, H.A., Bartczak, D., Fairbairn, N., Mcguire, E., Ardakani, M., Porter, A.E., and Kanaras, A.G., “Controlling the three-dimensional morphology of nanocrystals,” CrystEngComm 12(12), 4312 (2010).

[10] Bartczak, D., and Kanaras, A.G., "Diacetylene-Containing Ligand As a New Capping Agent for the Preparation of Water-Soluble Colloidal Nanoparticles of Remarkable Stability,” Langmuir 26(10), 7072-7077 (2010).

[11] Heuer-Jungemann, A., Harimech, P.K., Brown, T., and Kanaras, A.G., "Gold nanoparticles and fluorescentlylabelled DNA as a platform for biological sensing,” Nanoscale 5(20), 9503 (2013).

[12] Kymakis, E., Spyropoulos, G.D., Fernandes, R., Kakavelakis, G., Kanaras, A.G., and Stratakis, E., "Plasmonic Bulk Heterojunction Solar Cells: The Role of Nanoparticle Ligand Coating,” ACS Photonics 2(6), 714-723 (2015).

[13] Sun, Q.C., Ding, Y.C., Sagar, D.M., and Nagpal, P., "Photon upconversion towards applications in energy conversion and bioimaging,” in Prog. Surf. Sci. 92(4), Pergamon, pp. 281-316 (2017).

[14] Sun, L., Wei, R., Feng, J., and Zhang, H., “Tailored lanthanide-doped upconversion nanoparticles and their promising bioapplication prospects,” in Coord. Chem. Rev. 364, Elsevier, pp. 10-32 (2018).

[15] Sedlmeier, A., and Gorris, H.H., "Surface modification and characterization of photon-upconverting nanoparticles for bioanalytical applications,” Chem. Soc. Rev. 44(6), 1526-1560 (2015).

[16] Moskovits, M., “Surface-enhanced spectroscopy,” Reviews of Modern Physics 57(3), 783-826 (1985).

[17] Maier, S.A., [Plasmonics: Fundamentals and Applications], Springer US, New York, NY (2007).

[18] Paudel, H.P., Zhong, L., Bayat, K., Baroughi, M.F., Smith, S., Lin, C., Jiang, C., Berry, M.T., and May, P.S., "Enhancement of Near-Infrared-to-Visible Upconversion Luminescence Using Engineered Plasmonic Gold Surfaces,” The Journal of Physical Chemistry C 115(39), 19028-19036 (2011).

[19] Zhang, H., Li, Y., Ivanov, I.A., Qu, Y., Huang, Y., and Duan, X., "Plasmonic modulation of the upconversion fluorescence in NaYF4 :Yb/Tm hexaplate nanocrystals using gold nanoparticles or nanoshells.," Angewandte 
Chemie (International ed. in English) 49(16), 2865-8 (2010).

[20] Ma, X., Huh, J., Park, W., Lee, L.P., Kwon, Y.J., and Sim, S.J., “Gold nanocrystals with DNA-directed morphologies," Nature Communications 7, 12873 (2016).

[21] Niemeyer, C.M., “Nanoparticles, Proteins, and Nucleic Acids: Biotechnology Meets Materials Science,” Angewandte Chemie International Edition 40(22), 4128-4158 (2001).

[22] Zhang, Y., Lu, F., Yager, K.G., van der Lelie, D., and Gang, O., “A general strategy for the DNA-mediated selfassembly of functional nanoparticles into heterogeneous systems," Nature Nanotechnology 8(11), 865-872 (2013).

[23] Kanaras, A.G., Wang, Z., Bates, A.D., Cosstick, R., and Brust, M., "Towards multistep nanostructure synthesis: programmed enzymatic self-assembly of DNA/gold systems.,” Angewandte Chemie (International ed. in English) 42(2), 191-4 (2003).

[24] Young, K.L., Ross, M.B., Blaber, M.G., Rycenga, M., Jones, M.R., Zhang, C., Senesi, A.J., Lee, B., Schatz, G.C., et al., "Using DNA to design plasmonic metamaterials with tunable optical properties," Advanced Materials 26(4), 653-659 (2014).

[25] Tan, S.J., Campolongo, M.J., Luo, D., and Cheng, W., "Building plasmonic nanostructures with DNA,” Nature Nanotechnology 6(5), 268-276 (2011).

[26] Julin, S., Nummelin, S., Kostiainen, M.A., and Linko, V., "DNA nanostructure-directed assembly of metal nanoparticle superlattices.," Journal of nanoparticle research : an interdisciplinary forum for nanoscale science and technology 20(5), 119 (2018).

[27] Girard, M., Millan, J.A., and Olvera de la Cruz, M., "DNA-Driven Assembly: From Polyhedral Nanoparticles to Proteins,” Annual Review of Materials Research 47(1), 33-49 (2017).

[28] Ding, H.L., Zhang, Y.X., Wang, S., Xu, J.M., Xu, S.C., and Li, G.H., "Fe3O4@SiO2core/shell nanoparticles: The silica coating regulations with a single core for different core sizes and shell thicknesses," Chemistry of Materials 24(23), 4572-4580 (2012).

[29] Park, S.Y., Gibbs-Davis, J.M., Nguyen, S.T., and Schatz, G.C., "Sharp melting in DNA-linked nanostructure systems: Thermodynamic models of DNA-linked polymers,” Journal of Physical Chemistry B 111(30), 87858791 (2007).

[30] Gao, N., Chen, Y., Li, L., Guan, Z., Zhao, T., Zhou, N., Yuan, P., Yao, S.Q., and Xu, Q.H., "Shape-dependent two-photon photoluminescence of single gold nanoparticles,” Journal of Physical Chemistry C 118(25), 1390413911 (2014).

[31] Dong, H., Sun, L.-D., and Yan, C.-H., "Energy transfer in lanthanide upconversion studies for extended optical applications," Chemical Society Reviews 44(6), 1608-1634 (2015).

[32] Zhou, J., Liu, Q., Feng, W., Sun, Y., and Li, F., “Upconversion Luminescent Materials: Advances and Applications,” Chemical Reviews 115(1), 395-465 (2015). 